\title{
Levantamento sobre o uso de plantas medicinais com a terapêutica anticâncer por pacientes da Unidade Oncológica de Anápolis
}

\author{
OLIVEIRA, L.A.R.."; MACHADO, R.D.'; RODRIGUES, A.J.L. ${ }^{2}$ \\ ${ }^{1}$ Universidade Estadual de Goiás, UNuCET, Curso de Farmácia, ${ }^{2}$ Universidade Estadual de Goiás, UNuCET, \\ *leandra-almeida@hotmail.com Br 153, Km 98, Campus Henrique Santillo, CEP: 75001-970, Anápolis-Brasil
}

\begin{abstract}
RESUMO: Este estudo teve por objetivo conhecer o perfil dos pacientes em tratamento contra o câncer da Unidade Oncológica de Anápolis quanto ao uso de plantas medicinais. Foram entrevistados 59 pacientes $(42,12 \%$ da população estudada) por meio de questionários avaliativos enfatizando características sócio-demográficas e dados referentes à utilização de plantas medicinais. Evidenciou-se o uso indiscriminado de plantas medicinais entre os pacientes. A maioria dos entrevistados compartilha a opinião errônea de que plantas medicinais não fazem mal. A orientação sobre a forma de utilização das plantas ocorre, principalmente, pela informação de familiares ou amigos, e os profissionais da saúde muitas vezes são ignorados neste processo. Constatou-se que as plantas medicinais são utilizadas para o tratamento de enfermidades de baixa a alta gravidade, como o câncer. Dentre as 14 espécies usadas pelos pacientes com finalidade antineoplásica as mais mencionadas foram as popularmente conhecidas como noni, babosa, graviola e romã. Apesar de alguns estudos relatarem atividade antineoplásica ou quimiopreventiva para algumas espécies vegetais, muitas delas podem ser tóxicas ou apresentar potencial risco quando usadas concomitantemente ao tratamento convencional. Desta forma, observa-se que é preciso mais profissionais especializados para orientação sobre o risco de reações adversas e interações medicamentosas no que se refere ao uso de espécies vegetais e a terapêutica do câncer.
\end{abstract}

Palavras chaves: Tratamento do câncer. Plantas medicinais. Pacientes.

ABSTRACT: Survey on medicinal plants used with anticancer therapy by patients from Anapolis Oncology Unit. This study aims to survey the profile ofcancer patients in the Oncology Unit of Anápolis in relation to the use of medicinal plants. To evaluate socio-demographic characteristics and to retrieve data about on the use of medicinal plants, a questionnaire was filled by 59 patients, or $42.12 \%$ of the population under study. A widespread use of plants was found. Most of patients share the wrong idea that medicinal plants are not harmful. Guidance on the use of medicinal plants is given primarily by relatives and friends, and health professional are often ignored in this process. Among the 14 species used by patients with antineoplastic purpose, the most mentioned were popularly species known as "noni", "aloe", "soursop" and "pomegranate". Although some studies have reported chemopreventive or antineoplastic activity for some plant species, many may be toxic or show potential risk when used concurrently with conventional treatment. Thus, we can observe that there is a need for more specialized professionals for the guidance on the risk of adverse reactions and drug interactions in relation to the use of plant species and cancer therapeutics.

Keywords: Cancer treatment. Medicinal plants. Patients.

\section{INTRODUÇÃO}

Atualmente, o tratamento dos cânceres, em sua grande maioria, é considerado como um dos problemas mais desafiadores da medicina. De acordo com a localização, a extensão e o tipo de câncer pode-se optar por um ou por uma associação de tratamentos, dentre os quais se destacam a cirurgia, a radioterapia e a quimioterapia. Entretanto, observa-se cada vez mais a procura de tratamentos "alternativos e/ ou complementares" para o câncer por parte dos pacientes, junto aos médicos. Caso não seja atendido em sua solicitação, o paciente busca por conta própria ou com ajuda de familiares, amigos ou vizinhos, outras formas de tratamento focando 
o uso de espécies vegetais (Rang \& Dale, 2001; Araujo et al., 2007; Brasil, 2009).

Diversos trabalhos experimentais e epidemiológicos demonstraram que o consumo de algumas plantas pode promover ação quimiopreventiva e/ou antineoplásica. Nos países em desenvolvimento o amplo uso de terapias alternativas ou complementares, como o uso de plantas medicinais, se atribui à maior disponibilidade e acesso. Já nos países desenvolvidos o uso está impulsionado pela preocupação com os efeitos adversos dos fármacos sintetizados (OMS, 2002; Simões et al., 2002; Fukumasu et al., 2008).

Apesar do uso de plantas medicinais para tratamento, cura e prevenção de determinadas doenças ser uma das mais antigas formas de prática medicinal da humanidade e estar apoiada em um conhecimento consolidado por séculos de observação, planta medicinal não é sinônimo de inocuidade. Ao contrário do senso comum de que "medicamento natural se não fizer bem, mal não faz" a planta medicinal é um xenobiótico, ou seja, um produto estranho ao organismo com finalidades terapêuticas, que ao ser introduzido no organismo humano sofre biotransformação e pode, desta forma, gerar produtos tóxicos (Veiga Junior et al., 2005; Nicoletti et al., 2007).

$\mathrm{O}$ uso de plantas medicinais e/ou produtos à base de plantas medicinais como tratamento complementar, em concomitância ao tratamento oncológico convencional, pode ser perigoso, uma vez que os medicamentos antineoplásicos em sua maioria apresentam baixo índice terapêutico, ou seja, dose terapêutica muito próxima da dose tóxica. Uma preocupação adicional com o paciente oncológico é que este geralmente necessita receber vários outros medicamentos, além do quimioterápico, para minimizar as possíveis complicações do tratamento. Desta forma, o uso de plantas associado ao tratamento oncológico pode apresentar consequências indesejáveis, podendo mesmo, em alguns casos, comprometer a vida do indivíduo (Simões et al., 2002; Fukumasu et al., 2008).

No intuito de contribuir para o aumento do conhecimento nesta área, foi realizado um levantamento de dados para identificar a prevalência do uso de plantas medicinais por pacientes com câncer da Unidade Oncológica de Anápolis (UOA), conhecer as principais plantas medicinais utilizadas pela população estudada e seu padrão de uso. Esse estudo tem como perspectiva cooperar para a definição do quadro atual de utilização de plantas medicinais, e a partir daí facilitar a tomada de medidas que promovam o seu uso racional.

\section{METODOLOGIA}

Este estudo foi iniciado após aprovação do Comitê de Ética em Pesquisa da Associação de Combate ao Câncer em Goiás (ACCG) (Parecer n. 002/2011, datado de 14 de março de 2011). Desta maneira, a pesquisa atendeu aos requisitos da Resolução 196/96 e suas complementares do Conselho Nacional de Saúde (Brasil, 1996).

Os dados foram coletados na UOA, durante o mês de abril de 2011. A UOA é uma unidade ambulatorial de prevenção, diagnóstico e tratamento do câncer da ACCG que atende pacientes de Anápolis e toda região nordeste do Estado de Goiás. O hospital atende em média 140 pacientes com câncer por mês para os tratamentos de quimioterapia, radioterapia e cirurgia, entre outros (informação verbal) ${ }^{1}$. Foram entrevistados 59 pacientes, ou seja, um total de $42,14 \%$ da população disponível.

Participaram da pesquisa pacientes de ambos os gêneros, capacitados mentalmente, acima de 18 anos, que estavam em tratamento oncológico na UOA e que aceitaram participar da pesquisa de forma voluntária de acordo com o Termo de Consentimento Livre e Esclarecido.

A coleta de dados deste estudo ocorreu pela aplicação de um questionário de pesquisa (APÊNDICE 1) com auxílio do pesquisador e respondido individualmente pelos pacientes. Foram avaliadas as variáveis sócio-demográficas (gênero, idade, escolaridade, local de moradia) dos pacientes entrevistados e, principalmente, dados relevantes para pesquisa referente ao perfil de uso de plantas com propriedades medicinais.

A seleção dos pacientes foi realizada por meio do método não probabilístico, tendo como critério a disponibilidade do paciente em participar da pesquisa. A amostra foi selecionada de forma não-aleatória. Todos os indivíduos foram orientados quanto aos objetivos do estudo.

Os dados obtidos foram avaliados de forma qualitativa e quantitativa a partir de 59 questionários aplicados aos pacientes em tratamento. Para a avaliação quantitativa foi realizada uma análise estatística descritiva com auxílio do programa Microsoft Office Excel 2007.

\section{RESULTADOS E DISCUSSÃO}

\section{Dados sócio-demográficos}

Dos 59 pacientes entrevistados, $66,10 \%$ foram mulheres e $33,90 \%$ homens. A

\footnotetext{
${ }^{1}$ Dados fornecidos por Ana Paula Alves Peres Rodrigues (Responsável Técnica Geral da UOA), Nayara Lemes Guerra Cavalcante (Responsável pelo Setor de Quimioterapia) e por Lucimar Lopes de Andrade Costa (Responsável pelo Setor de Radioterapia).
} 
predominância feminina pode ser justificada pela maior disponibilidade das mulheres em responder ao questionário, pois estudos anteriores demonstram não haver diferença significativa entre o percentual de pacientes em tratamento do câncer dos sexos feminino e masculino (Brasil, 2008). Do total de casos novos de câncer no ano de 2006, 234.570 $(49,69 \%)$ foram do gênero masculino e 237.480 $(50,30 \%)$ do gênero feminino. (Brasil, 2009).

No que se refere à idade dos pacientes, $47,46 \%$ tinham mais de 59 anos. No entanto, segundo o Instituto Brasileiro de Geografia e Estatística (IBGE), no Brasil a população de pessoas com idade superior a 60 anos é somente $24 \%$ do total, o que demonstra maior incidência de câncer em pacientes idosos. Esse dado é compreendido visto que há uma relação direta entre a ocorrência de neoplasias e o processo de envelhecimento, fator esse associado à prolongada exposição aos fatores ambientais ou carcinógenos endógenos e ao seu efeito cumulativo (Belon, 2006; IBGE, 2008;).

No que diz respeito à escolaridade, $15,25 \%$ dos pacientes disseram ser analfabetos, 18,64\% disseram saber ler e escrever, 33,89\% possuía ensino fundamental incompleto/completo, $25,42 \%$ possuía ensino médio incompleto/completo e $6,77 \%$ com ensino superior incompleto/completo. Entre os pacientes entrevistados que alegaram ser analfabetos ou somente alfabetizados, a maioria $(85,01 \%)$ correspondia aos pacientes com mais de 58 anos. Esse resultado pode ser considerado previsível, em decorrência do acesso restrito dos idosos à educação formal no passado e também pelo fato dos programas de alfabetização implementados nas últimas décadas não terem sido capazes de saldar essa dívida educacional (IPEA, 2007).

Dos pacientes participantes da pesquisa, $44,07 \%$ disseram residir em Anápolis. Já 44,06\% afirmaram morar em outras cidades do Estado de Goiás e $11,86 \%$ em cidades de outros estados da Federação Brasileira. Cabe ressaltar que muitos destes pacientes se encontravam temporariamente em Anápolis devido ao tratamento.

Quando questionados sobre o tipo de tratamento realizado, $37,29 \%$ dos pacientes afirmaram realizar radioterapia, $25,42 \%$ quimioterapia, $27,12 \%$ faziam tanto o tratamento quimioterápico quanto radioterápico e $10,17 \%$ se submeteram a procedimento cirúrgico.

\section{UOA}

Utilização de plantas pelos pacientes da

Quanto uso de plantas medicinais, 83,05\% dos pacientes afirmaram fazer uso de algum tipo de planta medicinal, já $8,47 \%$ afirmaram ter deixado de usar plantas medicinais durante o tratamento do câncer e $8,47 \%$ não usavam plantas medicinais.
Estes dados são condizentes com estimativas nacionais que apontam $82 \%$ da população brasileira como consumidores de produtos à base de plantas medicinais (Rodrigues \& Simoni, 2010).

A busca por produtos naturais como método terapêutico está presente de forma marcante na cultura popular (Di Stasi, 2007). Muitos fatores têm contribuído para o aumento da utilização de plantas medicinais, entre eles, o difícil acesso da população à assistência médica e farmacêutica, o custo dos medicamentos industrializados, bem como a influência exercida pelos meios de comunicação para consumo de produtos vindos de fontes naturais (Simões et al., 1998; Veiga Junior et al., 2005).

Dos pacientes que usavam plantas medicinais $85,71 \%$ afirmaram sempre ter feito o uso deste recurso terapêutico, e 8,16\% iniciaram o uso após o diagnóstico ou início do tratamento do câncer. Esta busca por práticas complementares de saúde se deve principalmente a dois fatores: a insatisfação com o tratamento convencional e a afinidade pela utilização de produtos naturais (Jaconodino et al., 2008).

Quanto à utilização de plantas medicinais, proporcionalmente, as mulheres faziam maior uso $(87,18 \%$ das entrevistadas) em relação aos homens ( $75 \%$ dos entrevistados). Além disso, todas as pacientes mulheres que afirmaram não usar plantas medicinais, disseram já ter utilizado deste recurso em algum momento de suas vidas, mas abandonaram esta prática após o diagnóstico ou início do tratamento oncológico. O câncer, no entanto, não foi citado como o motivo da não utilização de plantas medicinais pelos homens.

Segundo Casarin et al. (2005) a frequência maior de uso entre as pacientes do sexo feminino comprova o maior envolvimento da mulher na busca pelo processo de cura, tanto na medicina dita alopática quanto nas práticas não alopáticas, tanto para ela quanto para sua família. A mulher é, portanto ao mesmo tempo usuária de práticas populares e agente de sua divulgação.

A utilização de plantas medicinais é comum a todos os níveis de escolaridade. Logo, não foi possível estabelecer uma correlação entre o grau de instrução e a utilização de plantas medicinais. Em estudo realizado entre 2005 a 2007, que aborda o uso popular de plantas medicinais no Rio Grande do Norte, o nível de escolaridade dos entrevistados não influenciou no uso de plantas medicinais. Neste estudo todos os entrevistados com nível superior faziam uso de produtos obtidos de plantas, ainda que raramente (Mosca \& Loiola, 2009).

A maioria $(55,10 \%)$ dos pacientes que usam plantas medicinais considera que as mesmas, por serem naturais, não fazer mal. Dentre os que acreditam que as plantas podem ter efeitos

Rev. Bras. PI. Med., Campinas, v.16, n.1, p.32-40, 2014. 
colaterais, $30,61 \%$ responderam que estas fazem menos mal que os medicamentos convencionais e $14,29 \%$ relataram que elas podem fazer tão mal quanto um medicamento convencional.

De acordo com os dados obtidos é preocupante o fato da maioria dos pacientes serem adeptos do mito "natural não faz mal", já que estudos demonstram o potencial de toxicidade para várias espécies, antes usadas sem restrições e de forma indiscriminada (Oliveira \& Gonçalves, 2006). Segundo Resener et al. (2006), este mito muitas vezes é estimulado pela publicidade que não faz referência aos possíveis riscos da utilização de plantas medicinais.

Observou-se entre os pacientes que o uso foi baseado no conhecimento popular das plantas, principalmente aquele passado de geração em geração, por amigos e vizinhos $(77,54 \%)$. Acrescido a este conhecimento popular, $18,36 \%$ afirmaram sofrer influências dos meios de comunicação como livros, televisão, jornais, revistas e internet. Houve ainda pacientes que receberam influência de outros pacientes em tratamento no hospital $(6,12 \%)$. Somente $2,04 \%$ receberam indicação médica.

A cultura popular colabora com o uso indiscriminado de plantas medicinais dentro do contexto da automedicação. Entende-se como automedicação o uso de medicamentos sem nenhuma intervenção por parte de um médico ou outro profissional habilitado, nem no diagnóstico, nem na prescrição, nem no acompanhamento do tratamento. $O$ fácil acesso às plantas medicinais incentiva a busca de "um possível tratamento" por custo mais acessível à grande parcela da população (Pereira, 2006; Nicoletti et al., 2007;).

Apesar da importância da orientação dos profissionais de saúde, somente 16,33\% dos pacientes afirmaram se informar com algum deles antes de utilizar uma planta medicinal. $O$ profissional mais citado foi o médico $(62,50 \%)$.

Dentre os pacientes que utilizavam plantas medicinais durante o tratamento do câncer, 22,45\% disseram sempre informar o médico, 4,08\% informavam às vezes e a maior parte $(73,47 \%)$ não informava. Estes dados são condizentes com estudo realizado entre 2000 e 2001, que trata do uso de plantas medicinais pelos pacientes com câncer de hospitais da rede pública de saúde em João Pessoa (PB) em que $79 \%$ dos pacientes não informavam ao médico sobre a utilização de plantas (Araujo et al., 2007). O nível de desconhecimento dos médicos mostra ser preocupante, já que deveriam estar cientes dos riscos e benefícios potenciais que podem surgir do uso destas duas modalidades diferentes de tratamento (Cheng et al.,2010).
Dentre as fontes disponíveis de recursos medicinais à base de plantas a maioria dos entrevistados disse adquirir as plantas que utilizavam em seu próprio quintal $(67,34 \%)$, em seu círculo de amizades ou de familiares $(44,08 \%)$ e em supermercados, mercados ou feiras $(30,60 \%)$. Lojas de produtos naturais $(10,02 \%)$ e farmácias $(2,04 \%)$ ainda são pouco procuradas, neste contexto. Outras formas de obtenção, como a coleta das plantas em seu ambiente natural, representaram $4,08 \%$.

Os quintais são uma das formas mais antigas de manejo da terra, fato esse que, por si só, indica sua sustentabilidade. Além disso, compatibilizam o acesso e a coleta de plantas constituindo sistemas adaptados às necessidades locais e geram muitos benefícios como a valorização da cultura e do conhecimento popular. (Amaral \& Guarim Neto, 2008; Bieski \& Cruz, 2005; Pasa et al. 2005).

Segundo Bieski \& Cruz (2005) boa parte da população dos países em desenvolvimento depende do conhecimento popular para a resolução dos seus problemas de saúde, devido às dificuldades de acesso a médicos e a medicamentos, ou a aspectos culturais da comunidade. Como visto, a busca de plantas medicinais em farmácias e lojas de produtos naturais é baixa, e o próprio quintal ou o dos amigos representa a principal fonte destes recursos medicinais, provavelmente em razão do baixo custo que representam. Os resultados observados estão em concordância com Vinagó et al. (2007) que também apontam os quintais ou amigos como fonte principal de plantas medicinais.

Dentre os pacientes que afirmaram usar plantas medicinais, $48,98 \%$ associaram seu uso ao tratamento do câncer e 51,02\% utilizavam para tratar outras enfermidades tais como problemas renais, gripe, infecções, enjôo, dores no estômago, inflamações, calmante, entre outros. Daqueles pacientes que utilizavam este recurso para tratar o câncer, $87,50 \%$ acreditavam que as plantas medicinais podem ajudar, $8,33 \%$ que podem atrapalhar e $4,17 \%$ que estas não interferem no tratamento convencional. Já dos pacientes que não utilizavam plantas medicinais para tratar o câncer, $56 \%$ acreditavam que as plantas medicinais podem ajudar, $12 \%$ que podem atrapalhar, $20 \%$ que estas não interferem e $12 \%$ não souberam ou não quiseram responder sobre o uso das plantas concomitantes ao tratamento do câncer.

Pode-se observar que os pacientes que fazem uso das plantas durante o tratamento oncológico, geralmente o fazem na perspectiva de que as mesmas possam contribuir para a melhora do seu quadro de saúde. Embora a maioria (56\%)

Rev. Bras. PI. Med., Campinas, v.16, n.1, p.32-40, 2014. 
dos pacientes que não utilizava plantas para tratar o câncer também acreditasse que estas possam auxiliar no tratamento, muitos justificaram não fazer o uso deste recurso por thes faltar ainda informações em relação ao uso, segurança e a eficácia destes recursos terapêuticos. Outros disseram que ainda não usavam, mas que pretendiam começar a usar.

Embora a maioria dos pacientes $(70,83 \%)$ que utilizava plantas para tratar o câncer ter afirmado notar melhora nos sintomas da doença, $12,5 \%$ disseram ter notado piora dos sintomas (Figura 1). Em estudo citado anteriormente (Araújo et al., 2007) todos os pacientes que usaram alguma planta medicinal afirmaram ter obtido melhora relativa dos sintomas com o uso das plantas medicinais e não ter apresentado efeitos adversos ou indesejáveis.

\section{Plantas medicinais utilizadas para o tratamento do câncer}

Alguns pacientes utilizavam plantas medicinais para controle dos efeitos colaterais do tratamento, como o uso do alecrim (Rosmarinus officinalis L.) para alívio do mal estar após as sessões de quimioterapia, o barbatimão (Stryphnodendron barbatiman Mart.) na cicatrização de feridas (câncer de pele) e a camomila (Matricaria chamomilla L.) para aliviar as queimaduras provenientes da radioterapia. Outros pacientes utilizavam deste recurso acreditando que as plantas medicinais poderiam curar o câncer ou colaborar para que isto ocorresse (Tabela 1). Para essa finalidade foram citadas 14 espécies, dentre as quais as mais mencionadas pelos pacientes foram as popularmente conhecidas como noni $(75 \%)$, babosa $(37,50 \%)$, graviola $(16,66 \%)$, e romã $(8,33 \%)$; as outras espécies representaram juntas $41,60 \%$ (Tabela 1 ).

Diversos trabalhos experimentais demonstraram que o consumo de algumas plantas pode promover ação quimiopreventiva e/ ou antineoplásica (Simões, et al.,2002; Fukumasu et al., 2008). Entretanto, o maior problema referese ao fato de que a divulgação inadequada destes dados à população pode promover um estímulo ao uso destas plantas. Como observado, dos pacientes que utilizavam plantas medicinais, quase a metade $(48,98 \%)$, almejava atividades anticancerígenas, no entanto, muitas plantas utilizadas, além do efeito quimiopreventivo proposto na literatura e antineoplásico podem apresentar efeitos tóxicos (Oliveira \& Gonçalves, 2006).

Faz-se necessário mais pesquisas direcionadas ao uso seguro de plantas medicinais e que estas informações cheguem de forma segura e clara aos pacientes. Para isto, é necessário que os profissionais de saúde estejam preparados para a orientação adequada ao paciente e que haja um cuidado por parte dos pesquisadores e da mídia, científica ou não, na divulgação das informações geradas.

\section{CONCLUSÃO}

Os pacientes fazem uso de plantas medicinais tanto para condições clínicas de baixo risco como para doenças graves.

A orientação sobre a forma de utilização das plantas ocorre, principalmente, pela informação de familiares ou amigos.

A maioria dos pacientes compartilha a opinião errônea de que plantas medicinais não fazem mal e não buscam orientação dos profissionais da saúde antes de utilizá-las

Há necessidade de mais profissionais especializados para orientação sobre o perigo de reações adversas e interações medicamentosas no que se refere ao uso de espécies vegetais e a terapêutica do câncer.

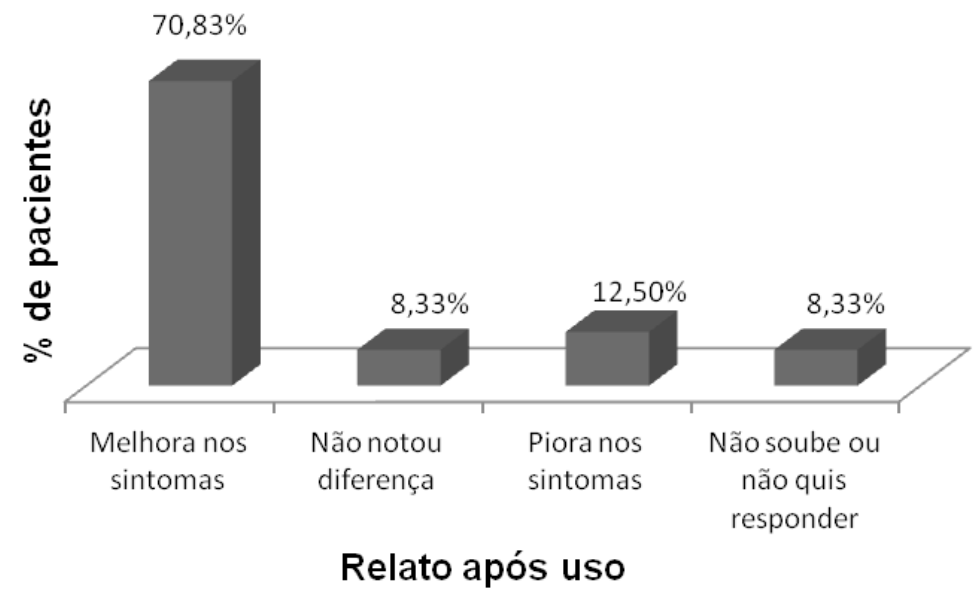

FIGURA 1. Resultados notados pelos pacientes da UOA, após a utilização de plantas medicinais. 
TABELA 1. Plantas medicinais citadas pelos pacientes da UOA para o tratamento do câncer. Modo de preparo e a forma de uso descrita pelos pacientes.

\begin{tabular}{|c|c|c|c|c|}
\hline $\begin{array}{l}\text { Nome da } \\
\text { planta }\end{array}$ & Nome Científico $^{2}$ & Família & Parte usada & Modo de preparo e Uso \\
\hline Araticum & $\begin{array}{l}\text { Annona } \\
\text { crassiflora Mart. }\end{array}$ & Annonaceae & Fruto inteiro & $\begin{array}{l}\text { Fruto in natura } \\
\text { Interno }\end{array}$ \\
\hline Aveloz & $\begin{array}{l}\text { Euphorbia } \\
\text { tirucalli L. }\end{array}$ & Euphorbiaceae & Folhas & $\begin{array}{l}\text { Infusão } \\
\text { Interno }\end{array}$ \\
\hline Babosa & $\begin{array}{l}\text { Aloe Vera (L.) } \\
\text { Burm. F. }\end{array}$ & Liliaceae & $\begin{array}{l}\text { Babosa sem os } \\
\text { espinhos } \\
\text { Mucilagem }\end{array}$ & $\begin{array}{l}\text { Processar no liquidificador a babosa sem } \\
\text { os espinhos com mel } \\
\text { Processar no liquidificador a mucilagem } \\
\text { sozinha ou com suco de uva } \\
\text { Mucilagem in natura } \\
\text { Pílulas da mucilagem com farinha de } \\
\text { trigo } \\
\text { Processar no liquidificador a mucilagem } \\
\text { com mel e aguardente } \\
\text { Processar no liquidificador a mucilagem } \\
\text { com mel, açafrão, } 1 \mathrm{~mL} \text { de creolina e } \\
\text { romã } \\
\text { Interno }\end{array}$ \\
\hline Burra-leiteira & $\begin{array}{l}\text { Sapium marmieri } \\
\text { Huber R. }\end{array}$ & Euphorbiaceae & Látex & $\begin{array}{l}\text { Dissolver o látex em água } \\
\text { Interno }\end{array}$ \\
\hline Cansanção & $\begin{array}{l}\text { Urera baccifera } \\
\text { (L.) Gaudich. Ex } \\
\text { Wedd }\end{array}$ & Urticaceae & Folhas & $\begin{array}{l}\text { Decocção } \\
\text { Interno }\end{array}$ \\
\hline $\begin{array}{l}\text { Castanheira- } \\
\text { do-Pará }\end{array}$ & $\begin{array}{l}\text { Bertholletia } \\
\text { excelsa Bonpl. }\end{array}$ & Lecythidaceae & $\begin{array}{l}\text { Ouriço (casca } \\
\text { que envolve as } \\
\text { castanhas) }\end{array}$ & $\begin{array}{l}\text { Triturar as cascas até obter a tenuidade } \\
\text { de um pó fino. Ingere com a comida. } \\
\text { Interno }\end{array}$ \\
\hline Cipó-santo & $\begin{array}{l}\text { Apodanthera } \\
\text { smilacifolia } \\
\text { Cong. }\end{array}$ & Cucurbitaceae & Látex & $\begin{array}{l}\text { Látex (Comprou pronto) } \\
\text { Interno }\end{array}$ \\
\hline Graviola & $\begin{array}{l}\text { Annona muricata } \\
\text { L. }\end{array}$ & Annonaceae & $\begin{array}{l}\text { Folhas } \\
\text { Frutos } \\
\text { Polpa }\end{array}$ & $\begin{array}{l}\text { Infusão das folhas e do fruto } \\
\text { Decocção } \\
\text { Folhas secas trituradas } \\
\text { Suco do fruto ou da polpa do fruto } \\
\text { Fruto in natura } \\
\text { Interno }\end{array}$ \\
\hline Jatobá & $\begin{array}{l}\text { Hymenaea } \\
\text { courbaril L. }\end{array}$ & $\begin{array}{l}\text { Leguminosae } \\
\text { Caesalpiniaceae }\end{array}$ & Casca & $\begin{array}{l}\text { Casca triturada com vinho branco } \\
\text { Interno }\end{array}$ \\
\hline Mangabá & $\begin{array}{l}\text { Hancornia } \\
\text { speciosa Gomes }\end{array}$ & Apocynaceae & Látex & $\begin{array}{l}\text { Látex diluído em água inglesa } \\
\text { Interno }\end{array}$ \\
\hline Noni & $\begin{array}{l}\text { Morinda citrifolia } \\
\text { L. }\end{array}$ & Rubiaceae & Fruto & $\begin{array}{l}\text { Processar no liquidificador o fruto com } \\
\text { suco de uva ou com vinho integral de } \\
\text { uva ou maça } \\
\text { Interno }\end{array}$ \\
\hline Pau d'óleo & Copaifera spp & $\begin{array}{l}\text { Leguminosae } \\
\text { Caesalpiniaceae } \\
\text { Fabaceae }^{1}\end{array}$ & Casca & $\begin{array}{l}\text { Maceração } \\
\text { Interno }\end{array}$ \\
\hline Romã & $\begin{array}{l}\text { Punica granatum } \\
\text { L. }\end{array}$ & Punicaceae & Fruto inteiro & $\begin{array}{l}\text { Processar no liquidificador o fruto com } \\
\text { suco de uva } \\
\text { Processar no liquidificador o fruto com } \\
\text { girassol e água } \\
\text { Interno }\end{array}$ \\
\hline Tiborna & $\begin{array}{l}\text { Synadenium } \\
\text { grantii Hoof }\end{array}$ & Euforbiaceae & Látex & $\begin{array}{l}\text { Látex diluído em água } \\
\text { Interno }\end{array}$ \\
\hline
\end{tabular}

Segundo Veiga Junior e Pinto (2002) todas estas classificações botânicas são utilizadas, porém, em uma classificação mais moderna Fabaceae Lindley dá lugar a Leguminosae Juss. 
APÊNDICE 1. Questionário de pesquisa

\section{Universidade Estadual De Goiás Unidade Universitária De Ciências Exatas E Tecnológicas Curso De Farmácia}

\section{ESTUDO DO CONSUMO DE PLANTAS MEDICINAIS PELOS PACIENTES COM CÂNCER DA UNIDADE} ONCOLÓGICA DE ANÁPOLIS (UOA)

Orientadora: Dra. Andréia Juliana Leite Rodrigues; Pesquisadoras: Leandra de Almeida Ribeiro Oliveira; Rúbia D’arc Machado.

\section{QUESTIONÁRIO DE PESQUISA}

1. Qual é a sua idade?

( ) 18 a 28

( ) 29 a 38

() 39 a 48

( ) 49 a 58

( ) mais que 58

2. Qual é o seu sexo?

( ) masculino

( ) feminino

3. Qual é sua escolaridade?

( ) analfabeto

() sei ler e escrever (alfabetizado)

( ) ensino fundamental incompleto

( ) ensino fundamental completo

( ) ensino médio incompleto

( ) ensino médio completo

() ensino superior incompleto

( ) ensino superior completo

( ) outro:

4. Em qual cidade você mora?

( ) Anápolis

( ) outra:

5. Que tipo de tratamento você faz na Unidade Oncológica de Anápolis?
( ) quimioterapia
( ) radioterapia
( ) cirurgia

6. Você usa plantas medicinais?

( ) $\operatorname{sim}$

( ) as vezes

( ) usava antes do câncer, não uso mais

( ) não

7. Em sua opinião, as plantas medicinais:

( ) por serem naturais não fazem mal

() fazem menos mal que os medicamentos convencionais

( ) podem fazer tão mal quanto um medicamento convencional

( ) podem fazer mais mal que um medicamento convencional

8. E com relação ao tratamento do Câncer, as plantas medicinais:

( ) podem ajudar o tratamento convencional

( ) podem atrapalhar o tratamento convencional

( ) não interferem no tratamento convencional
* Se sua resposta foi não para o uso de plantas medicinais, encerra-se o questionário.

9. Há quanto tempo você usa plantas medicinais?

( ) sempre usei plantas medicinais

( ) desde que fui diagnosticado(a) com câncer

( ) desde que comecei o tratamento do câncer

( ) outro:

10. Por que você usa plantas medicinais?

( ) porque acredito que as plantas medicinais podem me curar

( ) para amenizar os efeitos colaterais do tratamento

( ) para diminuir os sintomas da doença

( ) para auxiliar no tratamento convencional do câncer

( ) para tratar outras enfermidades, mas, não o câncer

( ) outro:

11. Quais plantas medicinais você usa?

12. Dentre estas plantas, você usa alguma(s) para "tratar" o câncer?

( ) Não

( ) Sim, qual (is):

13. Quem te recomendou usar esta(s) planta(s) medicinal(is)?

( ) amigos/vizinhos

( ) familiares

( ) médico

( ) enfermeiro

( ) farmacêutico

() meios de comunicação (televisão, jornais, revistas, internet)

( ) outro:

14. Você se informa sobre a planta medicinal com um profissional de saúde antes de usá-la?

( ) $\operatorname{sim}$

( ) as vezes

( ) não

* Se sua resposta foi não vá para a pergunta 16.

15. Com qual profissional de saúde você se informa sobre o uso de plantas medicinais?

( ) médico 
continuação...
( ) enfermeiro
( ) farmacêutico
( ) outro(s):

16. Onde você adquire as plantas medicinais que usa?

( ) com vizinhos, amigos ou familiares

( ) no quintal de casa

( ) no supermercado, mercados ou feiras

( ) em lojas de produtos naturais

( ) em farmácias

( ) outro(s):

17. O que você notou depois que começou a usar plantas medicinais?

( ) uma melhora nos sintomas

( ) não notei nenhuma diferença

( ) os sintomas pioraram

( ) outro:

18. Você informa ao seu médico quando usa plantas medicinais?

( ) $\operatorname{sim}$

() as vezes

( ) não
19. Por favor, descreva o preparo da planta medicinal que você usa:

Parte da planta que é utilizada:

Tipo de uso: ( ) interno ( ) externo

Em que quantidade (posologia):

Indicação:

Muito obrigada por participar desta pesquisa.

\section{REFERÊNCIA}

AMARAL, C.N.; GUARIM NETO, G. Os quintais como espaços de conservação e cultivo de alimentos: um estudo na cidade de Rosário Oeste (Mato Grosso, Brasil). Boletim do Museu Paraense Emílio Goeldi. Ciências Humanas, v. 3, n. 3, p. 329-341, 2008.

ARAÚJO, E.C. et al. Uso de plantas medicinais pelos pacientes com câncer de hospitais da rede pública de saúde em João Pessoa (PB). Revista Espaço para a Saúde, Londrina, v.8, n.2, p.44-52, jun. 2007.

BELON, A.P. Evolução e diferenciais sóciodemográficos da mortalidade por câncer de cólo de útero, mama feminina e próstata entre idosos no Estado de São Paulo de 1980 a 2000. 2006. 131f. Dissertação (Mestrado em Demografia)- Universidade de São Paulo. São Paulo.

BIESKI, I.G.C.; CRUZ, M.G. Quintais medicinais. Mais saúde, menos hospitais - Governo do Estado de Mato Grosso, Cuiabá, 2005.

BRASIL, Ministério da Saúde. Conselho Nacional de Saúde. Resolução n. 196, de 10 de outubro de 1996. Aprova diretrizes e normas regulamentadoras de pesquisas envolvendo seres humanos. Diário Oficial da União, Brasilia, DF, 16 de outubro de 1996.

BRASIL. Ministério de Saúde. Instituto Nacional de Câncer. Ações de Enfermagem para o Controle do Câncer Uma proposta de integração ensino-serviço. $3^{\text {a }}$. ed. atual. amp. Rio de Janeiro: INCA, 2008.

BRASIL. Ministério da Saúde. Instituto Nacional de Câncer. Estimativa/2010: incidência de câncer no Brasil. Rio de Janeiro: INCA, 2009.

CASARIN, S.T.; HECK, R.M.; SCHWARTZ, E. O uso de práticas terapêuticas alternativas, sob a ótica do paciente oncológico e sua família. Família, Saúde e
Desenvolvimento, v.7, n. 1, p.24-31, jan./abr.2005.

CHENG, $C$ et al. Evidence-Based Management of HerbDrug Interaction in Cancer Chemotherapy. Explore, v. 6, p. 324-329, 2010.

DI STASI, L.C. Plantas medicinais: verdades e mentiras: o que os usuários e os profissionais de saúde precisam saber. São Paulo: UNESP, 2007.

FUKUMASU, $\mathrm{H}$. et al. Fitoterápicos e potenciais interações medicamentosas na terapia do câncer. Revista Brasileira de Toxicologia, v. 21, n. 2, p. 49-59, 2008. INSTITUTO BRASILEIRO DE GEOGRAFIA E ESTATÍSTICA (IBGE). Population Projection of Brazil IBGE: Brazilian population aging is in an accelerated rhythm and will stop increasing in $\mathbf{3 0}$ years, 2008. Disponível em: <http://www.ibge.gov. br/english/presidencia/noticias/noticia_impressao. php?id_noticia=1272>. Acesso em: 25 de maio de 2011.

IPEA. Boletim de Políticas Sociais: acompanhamento e análise. Brasília: IPEA, n 13, 2007.

JACONODINO, C.B.; AMESTOY, S.C.; THOFEHRN, M.B. A utilização de terapias alternativas por pacientes em tratamento quimioterápico. Cogitare Enfermagem, v.13, n.1, p.61-66, 2008

MOSCA, V.M.; LOIOLA, M.I.B. Uso popular de plantas medicinais no Rio Grande do Norte, nordeste do Brasil. Revista Caatinga, v.22, n.4, p.225-234, 2009.

NICOLETTI, M.A. et al. Principais interações no uso de medicamentos fitoterápicos. Infarma, v.19, n. 1/2, p. 32-40, 2007.

OLIVEIRA, F.Q.; GONÇALVES, L.A. Conhecimento sobre plantas medicinais e fitoterápicos e potencial de toxicidade por usuários de Belo Horizonte, Minas Gerais. Revista Eletrônica de Farmácia, v. 3, n. 2, p. 36-41, 2006

ORGANIZACIÓN MUNDIAL DE LA SALUD. Estrategia de la OMS sobre medicina tradicional 2002-2005. Genebra, 2002. 
PASA, M.C.; SOARES, J.N. \& GUARIN-NETO, G. Estudo etnobotânico na comunidade de Conceição-Açu (alto da bacia do rio Aricá Açu, MT, Brasil). Acta Botanica Brasilica, v. 19, p. 195-207, 2005.

PEREIRA, J.R. et al. Riscos da automedicação: tratando o problema com conhecimento, 2006. Disponível em: <http://bvsms.saude.gov.br/bvs/premio_medica/pdfs/ trabalhos/mencoes/januaria_ramos_trabalho_completo. pdf> Acesso em: 28 maio 2011.

RANG, H.P.; DALE, M.M. Farmacologia. 4 ed. Rio de Janeiro: Guanabara Koogan, 2001.

RESENER, M.C.; SCHENKEL, E.P.; SIMÕES, C.M.O. Análise da qualidade de propagandas de medicamentos fitoterápicos disponibilizados em Santa Catarina. Acta Farmacêutica Bonaerense, v.25, n. 6, p. 583-589, 2006.
RODRIGUES, A. G.; DE SIMONI, C. Plantas medicinais no contexto de políticas públicas. Informe Agropecuário, Belo Horizonte, v. 31, n. 255, p. 7-12, 2010.

SIMÕES, C.M.O. et al. Plantas da Medicina Popular do Rio Grande do Sul, 5.ed., Porto Alegre: Editora da UFRGS, 1998. v.1.

SIMÕES, C.M.O. et al. Farmacognosia: da planta ao medicamento. 4.ed. Porto Alegre: ed. UFRGS, 2002.

VEIGA JUNIOR. V.F.; PINTO, A.C.; MACIEL, M.A.M. Plantas medicinais: cura segura? Química Nova, v.28, n.3, p. 519-528, 2005.

VINAGÓ, J.; VINAGÓ, J.A.; CRUZ-SILVA, C.T.A. Utilização de plantas medicinais pela população da região urbana de Três Barras do Paraná. Acta Scientiarum Health Science, v.29, n. 1, p. 51-58, 2007. 